\title{
La physiologie à la Haute-Ecole de Lausanne: le premier demi-siècle (1881-1932)*
}

\author{
Par Guy Saudan
}

Lausanne a très tôt rendez-vous avec la grande histoire de la physiologie, puisque Albrecht von Haller y publie dès 1757 son œuvre maîtresse, dominant la fin du $18^{\mathrm{e}}$ siècle, Elementa physiologiae corporis humani.

Haller à Lausanne: il s'agit là, en fait, d'une fort belle coïncidence. Ce sont, comme on le sait, l'Alpe et la flore de la région d'Aigle qui attirent l'éminent Bernois en Pays de Vaud; la réputation des imprimeurs de Lausanne et Yverdon fait le reste. En 30 ans, près de 50 volumes signés de son nom sortent des presses vaudoises ${ }^{1}$. Haller donne à la physiologie son statut de recherche indépendante et d'enseignement spécialisé ${ }^{2}$; mais Vaud, alors essentiellement rural et de plus soumis, ne recueille pas cet héritage-là, tirant par contre grand profit de l'influence politique du personnage dans le domaine de la santé publique. Ainsi Haller obtient-il du Sénat de Berne qu'il offre en 1766 au Dr Auguste Tissot le titre honorifique, premier du genre à Lausanne, de Professeur public en médecine à l' Académie, retenant l'intéressé au pays. Ainsi également, c'est avec l'appui de Haller que l'orthopédiste Jean-André Venel ouvre en 1778 à Yverdon la première école de sagesfemmes en Suisse.

Tissot, Venel, deux grands noms qui, avec celui du chirurgien Mathias Mayor, illustrent l'éveil médical vaudois des années 1750-1850, dans le sillage des Lumières, à l'aube de la médecine moderne et du Canton souverain ${ }^{3}$.

Le Canton de Vaud s'appartient en effet dès 1803: parmi les questions d'ordre médical, il lui faut tout d'abord traiter de l'organisation sanitaire et de la santé publique, avant de se préoccouper du développement et de la transmission de la science, soit de l'enseignement et de la recherche. L'Académie de Lausanne, héritée de Berne et de la Réforme, n'est qu'un séminaire de théologiens. Une première chaire laïque, en droit, y est introduite au début du $18^{\mathrm{e}}$ siècle; physique et mathématiques n'y sont

\footnotetext{
* Ce texte a fait l'objet d'une communication à la Journée annuelle de la Société suisse d'histoire de la médecine et des sciences naturelles, Lucerne, 9 octobre 1987, ainsi qu'à l'Institut de physiologie de Lausanne, 15 janvier 1988.
} 
autonomes que peu avant 1800; l'Académie elle-même n'est sécularisée qu'en 1837, par les Libéraux, et la Faculté des sciences se détache enfin de celle des Lettres en $1869^{4}$. C'est alors que la situation se précipite: en plein premier siècle de la médecine moderne et du Canton de Vaud, stimulé par les catalyseurs que représentent l'institution d'une Faculté de médecine à Genève (1876) et d'examens fédéraux (1877), le fédéralisme vaudois fait opter pour une Lausanne «ville d'études», dotant la Faculté des sciences d'une Section propédeutique médicale, en 1881. Une Faculté «complète» de médecine la suit de près, en 1890, transformant du coup l'Académie en Université de modèle allemand, grâce au généreux legs de Gabriel de Rumine (1888) et à la construction d'un nouvel Hôpital cantonal (1883).

La physiologie est dignement représentée à la Section propédeutique dès 1881, aux côtés de l'anatomie et embryologie, puis de l'histologie. Son premier demi-siècle à la Haute-Ecole (1881-1932) appartient à la dernière partie de l'âge d'or de la physiologie comme champ unitaire $(1840-1940)^{5}$, de la Belle Epoque aux Années folles, entre traditions française et allemande.

Chacun professeur 25 ans durant: Alexandre Herzen (1881-1906) - Russe d'origine, naturalisé Fribourgeois et en provenance de Florence -, puis le Français Maurice Arthus (1907-1932) - originaire d'Angers, en provenance de Marseille.

La dotation de la physiologie à Lausanne est extrêmement modeste: on se contente initialement d'une seule salle, logée dans d'anciennes casernes, à la Cité; d'un petit laboratoire ensuite, dans un ancien bâtiment des douanes, reconverti en Ecole de médecine (1888); enfin, au bout de près de 40 ans, on inaugure l'actuel Institut, rue du Bugnon, sous l'Hôpital.

La physiologie ne s'illustre-t-elle donc à Lausanne qu'avec les débuts de la médecine à l'Université?

L'activité des sociétés scientifiques vaudoises ne connaît de véritable essor que dans les années 1860. Un seul nom mérite absolument d'être retenu, avant que n'interviennent Herzen et Arthus: François-Alphonse Forel, professeur d'anatomie et de physiologie générales à la Faculté des sciences de l'Académie, de 1870 à 1895. Passionné de biologie, géophysique, météorologie, sismologie, préhistoire, Forel est le promoteur de la science des lacs, dite limnologie ${ }^{6}$.

Toujours est-il qu'en l'absence de candidat vaudois à la chaire de physiologie de médecine propédeutique, en 1881, le Conseiller d'Etat chef du Département de l'instruction publique se tourne vers les milieux de l'exil. Sur recommandation du Recteur de l'Université de Genève, le médecin 
allemand Karl Vogt, partisan de la gauche radicale en 1848, naturalisé à Genève, il fait appel à Alexandre Herzen (1839-1906), fils et homonyme du célèbre aristocrate et révolutionnaire russe émigré ${ }^{7}$, fervent disciple et assistant de l'allemand Moritz Schiff ${ }^{8}$ lui aussi expatrié pour motifs politiques. Athéisme, matérialisme, convictions libérales, dénominateurs communs de Vogt, Herzen et Schiff, n'ébranlent pas la détermination des autorités vaudoises: après tout, c'est le Recteur de Genève qui appuie Herzen. Ce dernier présente en outre une belle carte de visite: de Mueller et Magendie à Herzen, via Schiff, la filiation est directe.

Propagateur des idées de Schiff et continuateur de ses travaux, Herzen est son assistant à l'Institut des études supérieures de Florence, où il devient professeur au départ de son maître pour Genève en 1876. Spécialiste des fonctions nerveuses et digestives, pionnier de la psychologie physiologique, Herzen est un esprit cosmopolite, par son éducation, ses études, ses voyages; écrivant et parlant avec une égale facilité le russe, le français, l'allemand, l'italien et l'anglais, il connaît, par son père, Victor Hugo, Mazzini, Garibaldi.

Entraîné par son goût des généralisations, il étend ses intérêts à la philosophie, la pédagogie et la morale, où il révèle un grand rigorisme, propre d'ailleurs aux positivistes d'idées avancées de sa génération. Ainsi, au nom de la physiologie, lors de conférences publiques à Lausanne et Genève, intitulées Science et moralité, proscrit-il tout rapport sexuel avant vingt à vingt-deux ans, sous peine d'«étiolement» et d'«affaiblissement» frappant «à coup sûr et à perpétuité (...) non seulement l'individu mais sa progéniture». De même, il condamne explicitement le secret médical dans les cas d'atteintes syphilitiques ${ }^{9}$. Ces digressions mises à part, Herzen nous laisse notamment: Analyse physiologique du libre-arbitre humain (1870), Physiologie de la volonté (1874), La digestion stomacale (1886), Les conditions physiques de la conscience (1886), Fondements d'une psychophysiologie générale (1889) et Causeries physiologiques (1899). Il publie également à Lausanne les Mémoires physiologiques de son maître Schiff (1894-98) ${ }^{10}$.

Herzen est de ceux qui ont fait la Faculté de médecine de Lausanne, misant en particulier sur l'attraction d'étudiants étrangers, femmes russes surtout: doyen en 1906, l'année même de son décès, il est à la tête d'une Faculté forte de près de 500 étudiants - le sextuple de 1890 -, dont $85 \%$ d'étrangers, $69 \%$ de femmes; soit la moitié des étudiants de l'Université de Lausanne et le quart des candidats médecins de Suisse ${ }^{12}$.

Herzen subitement disparu des suites d'une intervention chirurgicale, la 
Faculté présente ex æquo deux des cinq candidats à sa succession: l'Italien Frédéric Battelli, futur gendre et successeur de Jean-Louis Prévost junior à Genève, et le Français Maurice Arthus, à Marseille ${ }^{13}$. Le second l'emporte et, parallèlement, Jean Larguier des Bancels est nommé professeur extraordinaire de physiologie des organes des sens ${ }^{14}$. Arthus (1862-1945) a été maître de conférences à la Sorbonne (1890), professeur de physiologie à Fribourg en Suisse (1896) - sa femme y est née -, chef de laboratoire à l'Institut Pasteur de Lille (1900), professeur de matière médicale et bactériologie à Marseille (1903). Son nom est attaché à la mise en évidence de l'anaphylaxie locale ou phénomène d'Arthus, en $1903^{15}$ : pierre dans le jardin de la sérothérapie et percée vers l'immunité et l'allergie. Fort de nombreux travaux sur la coagulation et les venins, Arthus se fait l'apôtre de la tradition expérimentale inaugurée par Magendie et Bernard, illustrée par Pasteur ${ }^{16}$. Excellent orateur, il at tire à ses cours une assistance variée et ne tarde pas à occuper une place prépondérante à la Faculté. Le psychiatre Auguste Forel, abstinent comme on sait, lui fait l'honneur d'une polémique par brochure interposée: Comme quoi l'alcool n'est pas un aliment, réponse au professeur Arthus ${ }^{17}$. A son arrivée à Lausanne, le nouveau titulaire de la chaire de physiologie obtient, du Chef du Département de l'instruction publique, promesse d'un institut ${ }^{18}$, inauguré en 1918, à mi-chemin de l'Ecole de médecine et de l'Hôpital cantonal. Le deuxième étage constitue le logement de fonction du professeur - innovation à l'Université de Lausanne -, service du laboratoire oblige, sur le modèle des instituts allemands ${ }^{19}$. De 1920 à 1924, Arthus est doyen, premier à assumer deux périodes bisannuelles consécutives. Concernant l'enseignement, il donne un Précis de physiologie (1921) qui devait faire autorité dans les pays francophones; il en va de même de son Précis de chimie physiologique (1895), édité 11 fois en 40 ans, et de son Précis de physiologie microbienne $(1921)^{20}$. Dans la préface à son ouvrage De l'anaphylaxie à l'immunité (1921 également), véritable morceau d'anthologie, Arthus propose quelques «directions» aux jeunes embrassant la carrière scientifique: l'accent est mis sur le sens critique, la méditation solitaire, la patience, l'esprit d'indépendance et d'originalité.

Conçu avant la Grande Guerre, sous la pression de la pléthore estudiantine étrangère, l'Institut des années 1920 se trouve délaissé par la clientèle de la Belle Epoque: Arthus instaure alors, dès 1922, en plein été, les Trois semaines de Lausanne, sorte de séminaire international de troisième cycle, où l'on accourt de France et de Belgique, d'Italie et de Pologne, et même du Canada, des Etats-Unis et d'Afrique ${ }^{21}$. 


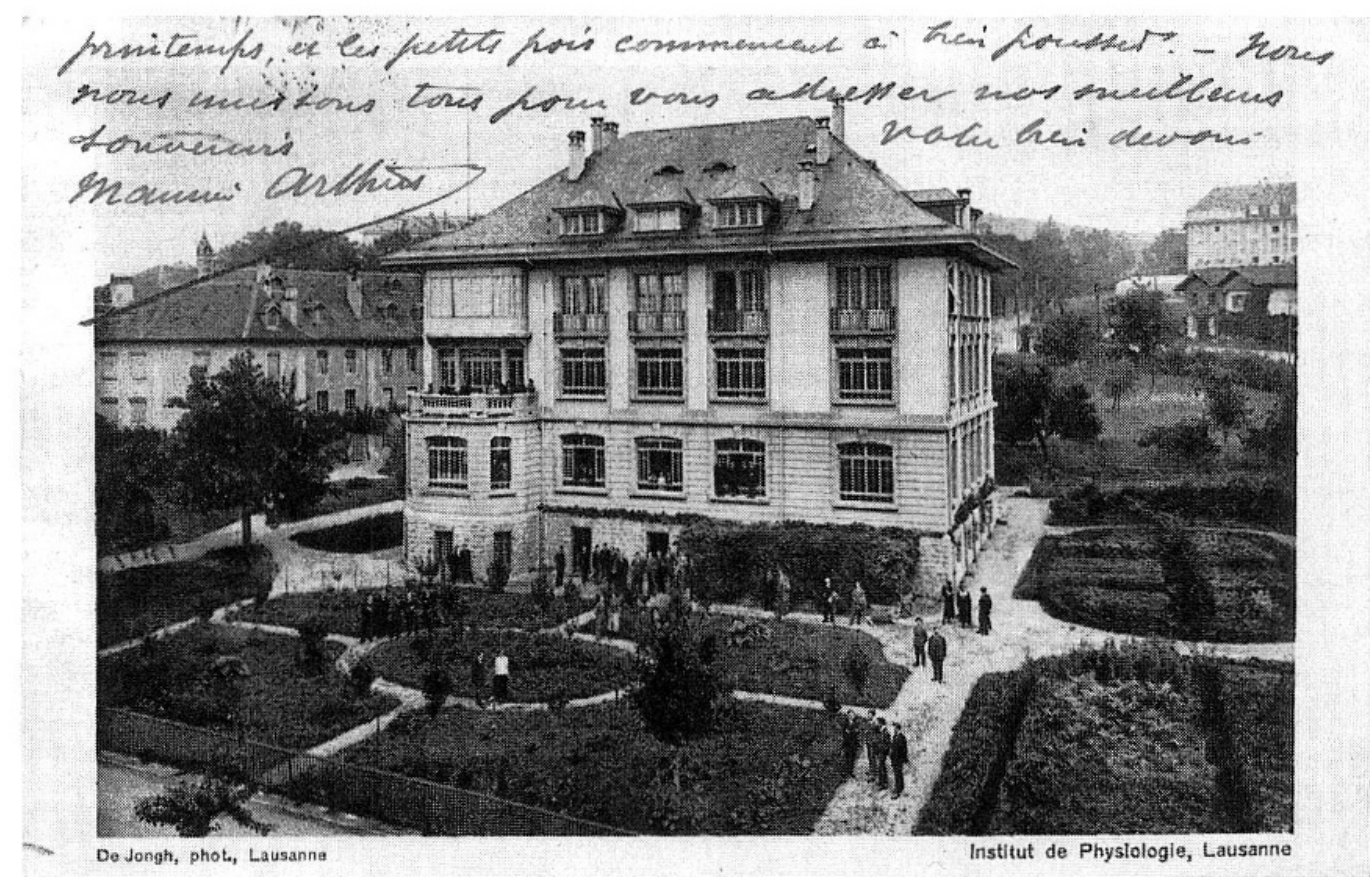

Fig. 1. L'Institut de physiologie en 1922

(Carte postale De Jongh, don Di Mattei, Faculté de médecine de Lausanne)

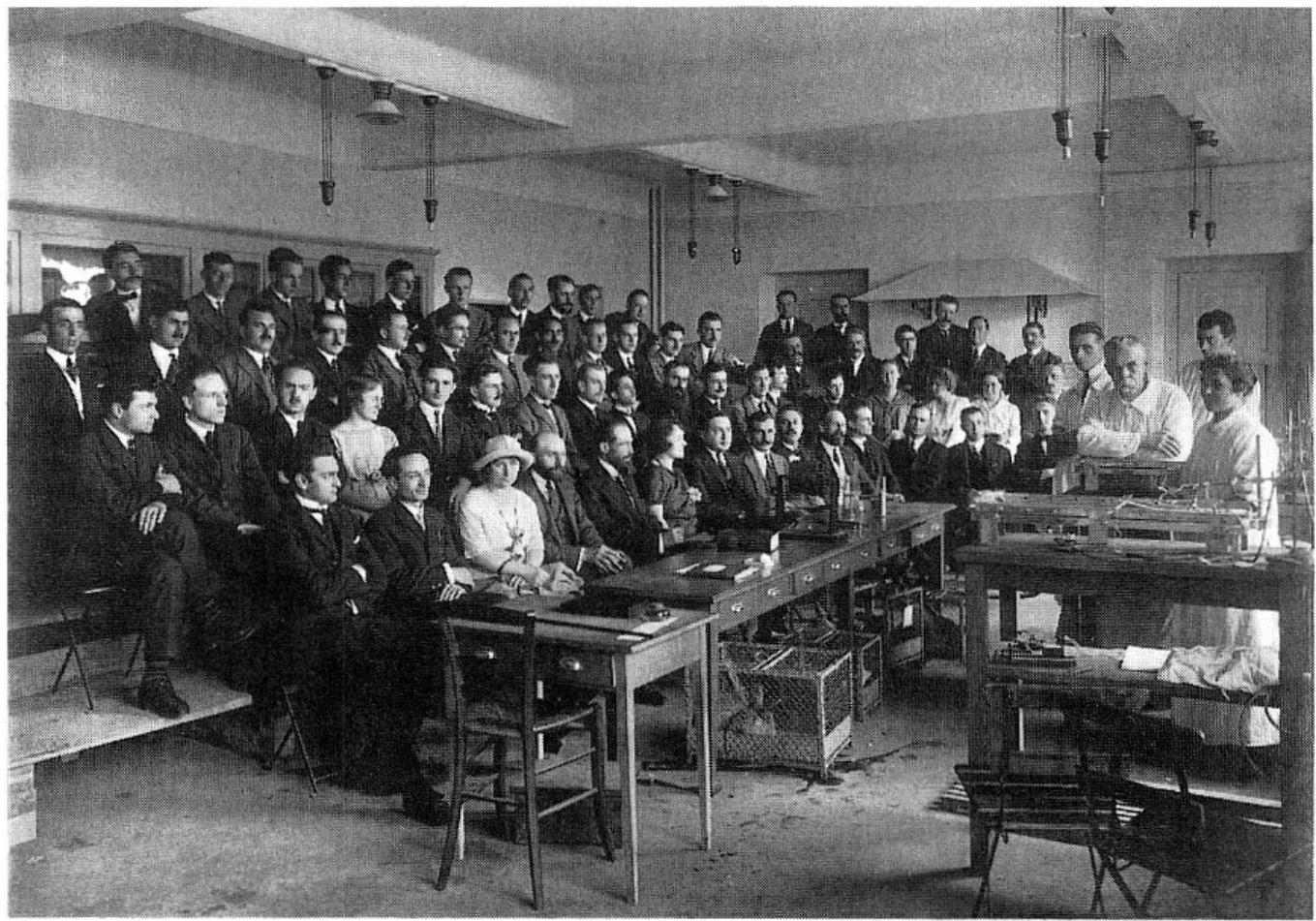

Fig. 2. Les Trois semaines de Lausanne, Institut de physiologie, $1^{\text {er }}$ étage, 1922

(Photo De Jongh, Musée de l'Elysée, Lausanne) 
A cette occasion, coïncidant d'ailleurs avec le soixantième anniversaire du maître, on édite des cartes postales de l'Institut, dont l'image passe ainsi les frontières, dans les Années dites folles.

Le premier demi-siècle de la physiologie à la Haute-Ecole de Lausanne, marqué par la création de la Faculté de médecine et le passage du simple laboratoire à l'Institut, se caractérise par un cosmopolitisme tant professoral qu'estudiantin: c'est un représentant de l'école française, Arthus, qui établit l'Institut, suivant les normes allemandes, pour une clientèle en majeure partie étrangère, slave.

Le contexte politique, local et international, paraît déterminant: fédéralisme vaudois, Suisse terre d'accueil des exilés démocrates, Europe d'avant 1914.

Une remarque méthodologique, pour conclure.

De notre sujet, il n'existe pour l'heure aucune étude topique, même partielle. De plus, il y a forte carence d'archives: pas même de rapports annuels à l'Institut. Par chance, quelques lettres et photographies nous sont parvenues par différents biais: à ce titre, nous remercions tout particulièrement le Professeur Pietro Di Mattei, de Rome, élève d'Arthus à Lausanne, ainsi que le Musée de l'Elysée et la Bibliothèque cantonale et universitaire, à Lausanne. Pour le reste, nous nous sommes adressé aux procès-verbaux, discours d'installation, ouvrages des protagonistes, ainsi qu'aux nécrologies et citations d'encyclopédies.

D'où, faute de mieux, une approche encore très fragmentaire.

Notes

1 Eugène Olivier, Médecine et santé dans le Pays de Vaud au XVIII siècle, 1675-1798, Concorde, Lausanne 1939, T. 2, Appendice I, p.902-904.

2 Georges Canguilhem, La constitution de la physiologie comme science, in: Etudes d'histoire et de philosophie des sciences, Vrin, Paris 1979 (1968), $4^{\mathrm{e}}$ éd., p. 226-273.

3 Antoinette Emch-Deriaz, Eugène Olivier et Jean Charles Biaudet, L'Eveil médical vaudois, 1750-1850: Tissot, Venel, Mayor, études réunies et présentées par Guy Saudan, préface du Professeur Michel Dolivo, Doyen de la Faculté de médecine de Lausanne, Université de Lausanne. Etudes et documents pour servir à l'histoire de l'Université de Lausanne XXI, 1987, 183 p.

4 Henri Vuilleumier, Notice historique et statistique sur l'Académie de Lausanne, in: Académie de Lausanne, Programme des cours, Lausanne, hiver 1878/79, p. 19 ss. 
5 Roderick E.McGrew, Encyclopedia of medical history, Macmillan Press, London 1985, «Physiology», p. 261.

6 Heinrich Buess, François-Alphonse Forel, in: Recherches, découvertes et inventions de médecins suisses, Ciba, Bâle 1946, p. 79.

7 «Alexandre Herzen», dossier ATS, Archives cantonales vaudoises.

8 Marc-A. Barblan, Moritz Schiff, in: Centenaire de la Faculté de médecine de l'Université de Genève, Médecine \& Hygiène, Genève 1978, p. 37-38.

9 Alexandre Herzen, Science et moralité, compte rendu sténographique des conférences données à Genève et à Lausanne en mars 1894, Payot, Lausanne 1894, p. 8.

10 C.P., Le Professeur Herzen, nécrologie, in: Revue médicale de la Suisse romande, 1906, p. 507-512.

B., Alexandre Herzen, professeur, in: La Patrie suisse, 5 septembre 1906, no 338, p. 208-209.

11 Guy Saudan, La Faculté de médecine (1890), in: De l'Académie à l'Université de Lausanne, 1537-1987, 450 ans d'histoire, Musée historique de l'Ancien-Evêché, Lausanne 1987, p. 220 .

Procès-verbaux des séances du Conseil de la Faculté de médecine de Lausanne, 15 mai 1893, p. 61.

12 La Faculté de médecine de Lausanne; Academia lausannensis, 1537-1987, dépliantaffiche, conception-réalisaton: Guy Saudan, Lausanne, Faculté de médecine, 1987.

13 Procès-verbaux des séances du Conseil de la Faculté de médecine de Lausanne, 14 novembre et 12 décembre 1906, p. 250-254.

Jean Posternak, La physiologie à la Faculté de médecine de Genève, in: Centenaire de la Faculté de médecine de l'Université de Genève, Médecine \& Hygiène, Genève 1978, p. 49-57.

14 Jean Larguier des Bancels, in: Livre d'or du $175^{\circ}$ anniversaire, 1806-1981, Belles-Lettres, Lausanne, Arts et Métiers, Lausanne 1981, p. 218.

15 Maurice Bariéty et Charles Coury, Histoire de la médecine, Fayard, Paris 1963, p. 728-736.

16 Maurice Arthus, Discours d'installation, séance d'installation du 7 novembre 1908, in: Discours d'installation, Université de Lausanne, Lausanne 1909, p. 145-155.

17 Auguste Forel, Comme quoi l'alcool n'est pas un aliment, réponse au professeur Arthus, Librairie nouvelle, Lausanne 1908, 15 p.

Suite de: Maurice Arthus, L'alcool est-il un aliment? Librairie nouvelle, Lausanne 1908, 16 p., où l'auteur fait le procès de l'alcool, en s'avouant néanmoins consommateur modéré.

18 Bâtiment pour la physiologie, rapport de la commission, in: Bulletin des séances du Grand Conseil du Canton de Vaud, 19 novembre 1913, p. 319.

19 Ibid., p.318. Cf. également: Exposé des motifs du projet de décret accordant au Conseil d'Etat un crédit extraordinaire de Fr.250000.- pour la construction d'un bâtiment de physiologie au Champ-de-l'Air, à Lausanne, Lausanne, automne 1913.

Hans-Heinz Eulner, Die Entwicklung der medizinischen Spezialfächer an den Universitäten des deutschen Sprachgebiets, Encke, Stuttgart 1970, p. 63.

20 Maurice Arthus' philosophy of scientific investigation (Preface to «De l'anaphylaxie à l'immunité», Paris 1921), translated from the French, with an introduction by Henry E. Sigerist, in: Bulletin of the history of medicine, XIV, Baltimore 1943, p. 368-372. 
21 Maurice Arthus, Lettre au Dr Pietro Di Mattei, de Rome, Lausanne 5 juin 1924, don du Professeur Di Mattei à la Faculté de médecine de Lausanne, 23 septembre 1986.

\section{Summary}

The coming of modern medicine to the region of Vaud owes much to the great Haller (1708-1777), who moreover published in Lausanne his Elementa physiologiae.

In the 19th century, with its combination of vaudois selfgovernment and the golden age of medicine, scientific societies were born, the Bernese Academy was secularized and, finally, the creation of a medical faculty led to the Haute-Ecole becoming a University in 1890 .

A chair of medical physiology was inaugurated in 1881 by Alexandre Herzen (1839-1906), a student of Moritz Schiff, son and namesake of the famous Russian revolutionnary aristocrat, who pioneered physiological psychology as well as specializing in stomach research.

Herzen's successor, the Frenchman Maurice Arthus (1862-1945), occupied the chair from 1907 to 1932 . He is known for discovering the phenomenon that bears his name, and for his work on coagulation and venoms. It was during this period that the "laboratoire" became an "institut".

\section{Guy Saudan}

Historien

3, chemin Ecureuils

CH-1012 Lausanne/Pully 